non-binding fraction and the composition of amniotic fluid altered accordingly. This is apparently so.

Since affinity chromatography is more complicated than conventional AFP assay it will probably be used on selected samples. If the method proves as reliable as suggested here, an appropriate strategy would be to lower the cut-off value to three standard deviations above the mean total AFP concentration and subject all samples with concentrations above this value to con-A-binding studies. Theoretically, this would give a high incidence of detection of open spina bifida with a specificity of nearly $100 \%$.

I am grateful to Sandra Brown and Lilias Barron for excellent technical help. The work was supported by a grant from the Medical Research Council.

${ }^{1}$ Ruoslahti, E, et al, International fournal of Cancer, 1978, 22, 515.

2 Smith, C J, et al, British Medical fournal, 1979, 1, 920.

3 Ruoslahti, E, et al, British Medical fournal, 1979, 2, 768

Brock, D J H, British Medical Bulletin, 1976, 32, 16

5 Brock, D J H, Lancet, 1976, 2, 345.

\section{Cyclical variations in mood in normal women taking oral contraceptives}

A significant number of women taking oral contraceptives develop pathological changes in their mood over a period of several months. ${ }^{1}$ Little, however, is known of the mood changes within the menstrual cycle of normal women taking oral contraceptives.

\section{Subjects, methods, and results}

Twelve volunteers were studied. All were healthy women aged between 22 and 30 . They were all taking oral contraceptives containing not more than $30 \mu \mathrm{g}$ of ethinyloestradiol per tablet and were happy with this method of

$\longrightarrow$ Mean scores Exponentially smoothed mean scores

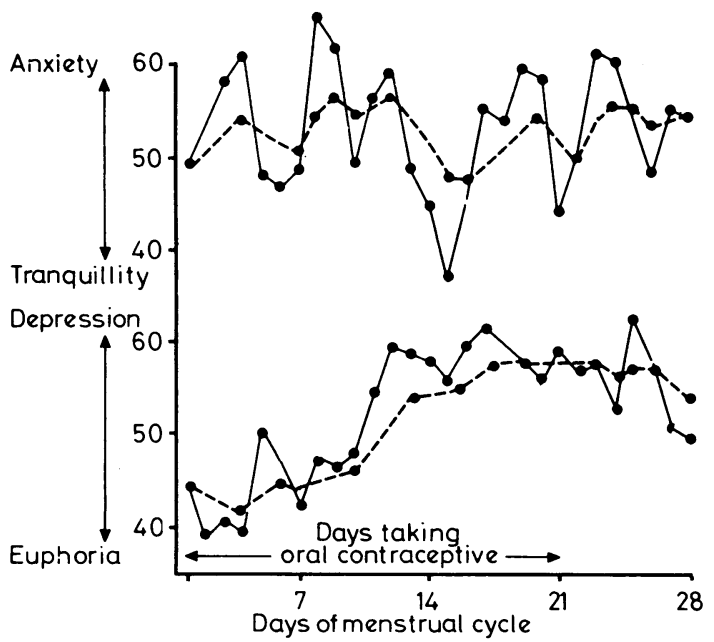

Variation of mean mood and anxiety level scores during menstrual cycle.

contraception. None had changed the brand of their oral contraceptive within three months of the start of the study. None had any history of mental illness. Each recorded an indication of her mood and affect every evening for 30 days. This was done by using visual analogue self-rating scales. ${ }^{2}$ These were forms, one for each day, on which were two $10-\mathrm{cm}$ lines separating statements describing extremes of mood (euphoria to depression) and anxiety level (tranquillity to anxiety). Before retiring for the night the subjects marked each of the lines in the position between the two sets of statements that best described their state of mind at the time. Subsequently, by measuring the position of the mark on the lines, numerical scores from zero to 100 for depression and anxiety for each subject each day were produced. To minimise any bias effects each woman started keeping records on a different day in the menstrual cycle and the records from the first two days for each woman were not analysed. The results from the group as a whole were pooled, and mean scores for depression and for anxiety for each day of the menstrual cycle, counting day 1 as the first day on which an oral contraceptive pill was taken, were calculated. The mean scores were then analysed by computer, calculating exponentially smoothed scores for each day and using Cembrowski et al's $s^{3}$ modification of Triggs's technique for trend detection. There was a steady increase in mean depression scores while the subjects were taking oral contraceptives. Triggs's technique showed that when the depression scores were smoothed so that the exponential mean corresponded to a moving average containing seven observations this trend was significant $(P<0.05)$ from days 11 to 19 of the cycle. The anxiety self-rating scores did not show any significant trend throughout the cycle.

\section{Comment}

The steady increase of the self-rating scores for depression during the menstrual cycle is consistent with the theory that depression associated with oral contraception has a metabolic basis. The metabolic lesion may be a functional deficiency of pyridoxine. ${ }^{4}$ Thus it would be of interest to investigate the effect, if any, that pyridoxine has on these cyclical mood changes. There was no tendency for anxiety selfrating scores to increase towards the end of the cycle, and this would support the use of oral contraceptives for the treatment of premenstrual tension.

I thank the volunteers for their co-operation in this study.

A listing of the computer program in BASIC used for the calculation of Triggs's statistic and for exponential smoothing is available from the author.

${ }^{1}$ Herzberg, B N, Johnson, A L, Brown, S, British Medical fournal, 1970, 4, 142.

${ }^{2}$ Zealley, A K, and Aitken, R C B, Proceedings of the Royal Society of Medicine, 1969, 62, 993.

${ }^{3}$ Cembrowski, G S, et al, Clinical Chemistry, 1975, 21, 1396.

4 Adams, P W, et al, Lancet, 1973, 1, 897.

(Accepted 24 September 1979)

Department of Biochemistry, Royal Infirmary, Glasgow G4 OSF A R W FORREST, MRCP, DOBSTRCOG, senior registrar

\section{How to evaluate papers given at medical meetings: Use of the SPEAKER Index}

The objective finding that fewer than half the speakers at a surgical meeting were able to communicate effectively ${ }^{1}$ confirms subjective experience that the standard of oral presentations at medical meetings is disappointingly low. There are many reasons why speakers communicate poorly. Two are interrelated: few doctors are taught how to communicate the results of their work (whether by the spoken or the written word), and most doctors therefore evaluate talks subjectively rather than objectively. In teaching doctors how to speak effectively, I emphasise the need for objective evaluation of oral presentations, and one result of this emphasis has been the development of the SPEAKER Index.

\section{The Index}

The SPEAKER Index is an aid to evaluating the performance of speakers. The mnemonic acronym SPEAKER refers to the initial letters of the seven primary performance characteristics that are listed in part 1 of the Index (see figure). These primary characteristics may be rated from low to high on a five-point scale contained on the first side of a single sheet; the overall rating may be displayed numerically, by calculating a mean score, or graphically, by joining numerical points. The other side of the sheet, which is given over to part 2, lists the many secondary characteristics that can be subsumed under the primary characteristics. These secondary characteristics may be 